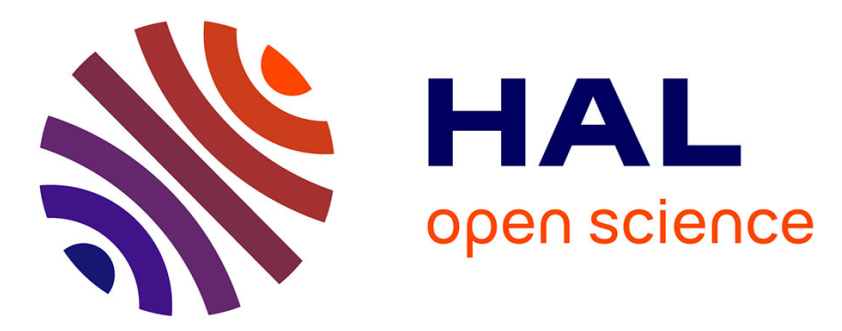

\title{
Surface morphology features of point contact gas sensors based on Cu-TCNQ compound
}

D. Harbuz, A. Pospelov, V. Gudimenko, A. Konotop, P. Mateychenko, D. Kowalska, E. Faulques, G.V. Kamarchuk

\section{- To cite this version:}

D. Harbuz, A. Pospelov, V. Gudimenko, A. Konotop, P. Mateychenko, et al.. Surface morphology features of point contact gas sensors based on Cu-TCNQ compound. Molecular Crystals and Liquid Crystals, 2021, 718 (1), pp.25-35. 10.1080/15421406.2020.1861518 . hal-03356238

\section{HAL Id: hal-03356238 \\ https://hal.science/hal-03356238}

Submitted on 18 Nov 2021

HAL is a multi-disciplinary open access archive for the deposit and dissemination of scientific research documents, whether they are published or not. The documents may come from teaching and research institutions in France or abroad, or from public or private research centers.
L'archive ouverte pluridisciplinaire HAL, est destinée au dépôt et à la diffusion de documents scientifiques de niveau recherche, publiés ou non, émanant des établissements d'enseignement et de recherche français ou étrangers, des laboratoires publics ou privés. 


\section{Surface morphology features of point contact gas sensors based on $\mathrm{Cu}$-TCNQ compound}

D.A. Harbuz ${ }^{\mathrm{a}}$, , A.P. Pospelov ${ }^{\mathrm{a}}$, V.A. Gudimenko ${ }^{\mathrm{b}}$, A.P. Konotop ${ }^{\mathrm{b}}$, P.V. Mateychenko ${ }^{\text {c }}$, D.A. Kowalska ${ }^{\text {d }}$, E. Faulques ${ }^{\mathrm{e}}$, G.V. Kamarchuk ${ }^{\mathrm{b}}$

${ }^{a}$ National Technical University "Kharkiv Polytechnic Institute", Kharkiv, Ukraine; ${ }^{b}$ B. Verkin Institute for Low Temperature Physics and Engineering, National Academy of Sciences of the Ukraine, Kharkiv, Ukraine; ${ }^{c}$ Institute for Single Crystals, National Academy of Sciences of the Ukraine, Kharkiv, Ukraine; ${ }^{d}$ Institute of Low Temperature and Structure Research, Polish Academy of Sciences, Wroctaw, Poland; ${ }^{e}$ Institut des Matériaux Jean Rouxel (IMN), Université de Nantes, CNRS, Nantes, France

e-mail: zeyxes@gmail.com

D.A. Harbuz - 0000-0002-5241-9714

A.P. Pospelov - 0000-0002-5136-9268

V.A. Gudimenko - 0000-0001-6924-3818

A.P. Konotop - 0000-0003-0902-9224

P.V. Mateychenko - 0000-0002-4074-195X

D.A. Kowalska - 0000-0001-8180-653X

E. Faulques - 0000-0002-7761-8509

G.V. Kamarchuk - 0000-0002-1105-8019 


\section{Surface morphology features of point contact gas sensors based on Cu-TCNQ}

\section{compounds}

The 7,7,8,8-tetracyanoquinodimethane (TCNQ) radical anion salt compound was used as the sensitive material of gas point contact sensors. The phase composition and surface morphology of the obtained sensor films were established. It was found correlation between surface morphology of the point-contact transducer and definite type of the sensor response curve observed under action of the human breath gas. If the sensor sample is formed from numerical crystals of the same or similar sizes having a uniform shape, similar to a shell, and clearly defined external borders it indicates to the creation of defect-free Yanson point contacts in the process of soft electrochemical synthesis. As a result, the surface of the sensor is a complex multistructure of a numerous number of Yanson point contacts, which generates a large output signal from the sensor in response to the action of the analyte. When crystals of $\mathrm{Cu}$-TCNQ compound forming samples under investigation have lower density, large size, distorted and full of fractures boundaries, it prevents the formation of high-quality point contacts. These morphologic peculiarities are typical for samples with low level of response signal. In the case of extremely low density of $\mathrm{Cu}$-TCNQ crystals observed in the sample surface the sample does not show any response to the action of the human breath gas.

Keywords: gas sensor; Yanson point contact; breath analysis; metal-polymers;

electrochemical deposition;

\section{Introduction}

Studies of nanostructured objects have become increasingly important in recent years due to the wide possibilities of using such materials [1]. The area of their application can vary greatly - from construction materials to materials for electronics and medicine. Gas nanosensors are one of the important fields in which they are used [2]. In advanced nanosensors, promising properties have been demonstrated by carbon nanotubes [3, 4]. However, despite the indisputable advantage of carbon-nanotube-based sensors, including ultra-high sensitivity to the analyte [5] and the possibility of functionalization [6], they also have a noticeable disadvantage - the long relaxation time after exposure to a gas agent [7].

One of the most perspective applications of nanosensors is their use as sensors for analyzing human breath gas [8]. They have already shown their high efficiency as tools for early non-invasive detection of lung cancer, which is not an easy task for other methods used in this area [9]. A peculiarity of breath gas used as analyte is a very high number of specific components, as well as their ability to react with each other over short periods of time. The various components are of different nature and origin, including gastrointestinal tract, lungs, oral cavity, or various exogenous factors, such as smoking and the environment state. The number of compounds in the breath gas may amount to 2000 [10]. Some of these components carry information about the metabolic state of the human body. The ability to detect these components, differentiate one from another, and associate them with various pathogens or conditions, is a key to successful implementation of these sensors in a wide medical practice [11].

Currently, the so-called electronic noses are widely used as sensors for human breath gas [12]. They present a set of several conducting structures, mainly nanostructures, with various electrophysical properties and, therefore, different response parameters to various gas components. However, their significant disadvantage is the complexity and long duration of the processing of the results, which is related to the capabilities of modern computer technology 
[13]. As a result, such sensors are usually able to identify about 20-30 components of the gas mixture in real conditions. This fact limits significantly the area of their possible application.

In recent years, an alternative and fundamentally different approach to the sensor analysis of complex gas mixtures has manifested itself to be more powerful [14]. Its main idea is to use gas sensors based on Yanson point contacts. Point contacts are the main tool of the Yanson point contact spectroscopy, which is based on the fundamental theory and wide practice of studying fine structures of condensed phases [15]. The Yanson point contact spectroscopy has been studied well and is widely used because it's relatively easy to use and, most importantly, makes it possible to determine with high accuracy the electron-phonon interaction function [16]. It has turned out that Yanson point contacts, thanks to their unique properties, are a promising basic element of nanostructured gas sensors [17].

A large group of chemical gas sensors are devices based on their ability to change their electric resistance in response to the action of a gas medium. In the zeroth-order approximation, this feature reflects the change in the cross-section area of the sensor conducting channel due to the modification of its surface layer. It thus becomes clear that the greater the surface-to-volume ratio of the sample, the higher the sensitivity of the gas sensor. Yanson point contacts, in their turn, are electrical contacts between two massive electrodes formed in a space smaller than or comparable to the mean free path of charge carriers in the material. Hence the small size of such contacts, normally a few nanometers, which results in a large surface-to-volume ratio for the conductor and, therefore, in a high sensitivity of the sensors. But geometrical factor is not the main cause of the extreme possibilities of the point-contact nanosensors [18]. Their extra performance are determined, first of all, by the basic properties of Yanson point-contacts. Here are some relevant examples. The potential distribution in the Yanson point contact is quite specific with the drop observed directly in the contact area. It means, that the entire electric field is concentrated in the point contact and the changes of its electric resistance affect the changes of the resistance of the entire system $[14,18]$. Due to the fact that the thermal energy emission caused by inelastic scattering of electrons does not occur in the contact area, it is possible to reach a very high current density in these objects - up to $10^{7} \mathrm{~A} / \mathrm{cm}^{2}$ at room temperature [17]. Under the action of a high-density current, adsorbed gas molecules desorb from the contact surface in a few seconds or minutes, with the surface returning it to its original state without any additional processing.

In the previously published works, we showed the high efficiency of point-contact gas sensors based on tetracyanoquinodimethane compounds in the studies of human breath gas aimed at differentiating among the various strains of Helicobacter pylori bacterium [19, 20]. The sensors used in these works were selected from the manufactured batches of sensors according to a special procedure using the criteria described in [21]. In these batches of sensors, along with selected high-quality samples, there were also sensors with a low level of output signal for the action of human breath gas. In order to understand the reasons for the appearance of differences in the response signals of samples of point-contact sensor arrays based on TCNQ compounds to the action of human breath gas, the task was set to study the state of the surface of the obtained point-contact sensors, since the surface of the sensor structure has a significant effect on its operation. Based on this, the purpose of this research was to study the surface morphology of various point-contact sensors and to make an appropriate analysis to identify the possible relationship of this factor with the nature of the sensory response to the action of human breath gas. 


\section{Materials and methods}

Anion-radical salt 7,7,8,8-tetracyanoquinodimethane (TCNQ) was used as a sensitive material to obtain a point-contact sensor array. The reason for this choice is the features of the crystal structure of this material and its electrophysical properties. Crystals of these salts grow mainly in the form of nanoneedles, nanotubes or nanopellets with a characteristic packing of molecules in stacks $[22,23]$. Yanson point contacts are formed in the contact areas of these crystals. So we used the Chubov technique, which is applied in the Yanson point contact spectroscopy to obtain similar nanostructures [24].

During the study, two types of objects were obtained - functional and model samples. While functional sensors were used directly to study their response to the action of a gas medium and determine the relationship between the surface morphology and the response parameters, model samples were used to determine the dependence of the morphology and structure on the deposition regimes.

Functional samples were obtained by means of combined electrochemical deposition of the salt from a saturated solution of acetonitrile in constant voltage mode in accordance with the procedure described in Ref. [23]. Model samples were obtained in the way described below. At first, copper was thermally deposited on a single-crystal silicon substrate with a (100) surface orientation. The thickness of the obtained copper film was $300 \mathrm{~nm}$. Then, the obtained samples were subjected to electrochemical and combined electrochemical deposition in various regimes, similar to and different from those used to obtain functional sensors.

During the deposition, the TCNQ salt reacted with copper on the substrate, forming the $\mathrm{Cu}-\mathrm{TCNQ}$ compound by the direct oxidation reaction [25]:

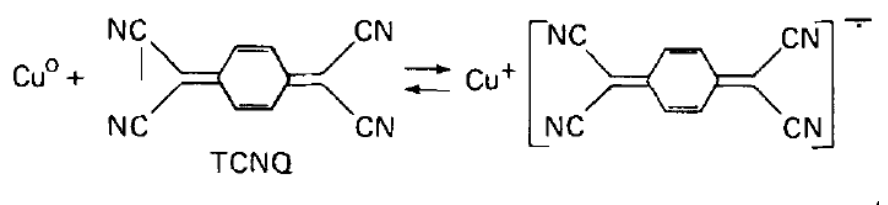

The use of copper is due to the fact that this material has a high specific conductivity and is quite technological, which is important for the creation of Yanson point contacts. In addition, copper salts based on TCNQ compounds are well studied and possess the necessary physicochemical properties for the creation of point-contact sensors. For model samples of sensors, the thickness of the copper film was determined in the process of its thermal deposition using a piezoelectric crystal. For functional samples of sensors, the thickness of the copper foil was standard $(0.5 \mathrm{~mm})$, since for their production, factory textolite substrates for electronics were used.

One of the two phases of polymorphic compounds $\mathrm{Cu}$-TCNQ could be formed during the reaction [14]. The phase composition of the obtained samples was determined using $\mathrm{X}$-ray diffraction.

X-ray diffraction studies were carried out using a PANalitycal X'Pert Pro X-ray diffractometer. A $\theta-2 \theta$ scan was performed in the $2 \theta$ range of $10-80^{\circ}$ with a scan speed of $3^{\circ} \mathrm{min}^{-1}$ in the $K_{\alpha}$ radiation of copper. The studies were carried out on the basis of the model samples; single crystal silicon with a surface orientation of (100) was used as the substrate. The surface morphology of the samples was studied using a scanning electron microscope A JSM-6390LV. 
To record the sensor response to the action of breath gas in real-time regime, we used a portable measuring system created by our research group, which was connected to a personal computer with the original software SensorResponce made in B. Verkin Institute for Low Temperature Physics and Engineering. The sensor response was recorded by measuring the voltage drop on a precision $1 \mathrm{M} \Omega$ resistor connected in series with the sensor. The output was amplified by 10 times. The data obtained using the portable measuring complex were checked with a Keithley 2100 multimeter. More details can be found in Refs. [19, 21].

To measure the sensor response to the action of human breath gas, a specific measurement technique similar to the one used in medical studies was implemented [20, 26]. Measurement of the temporal dependence of the sensor conductance was performed during the action of a given gaseous medium. A typical sensor response to the breath gas is shown in Fig. 1. There are curves obtained under action of the breath gas of the same person for sensors from 3 different banches. Parameters used to analyze the response curve are also presented here. Various metabolic states of the human body can be determined using different combinations of these parameters [27].

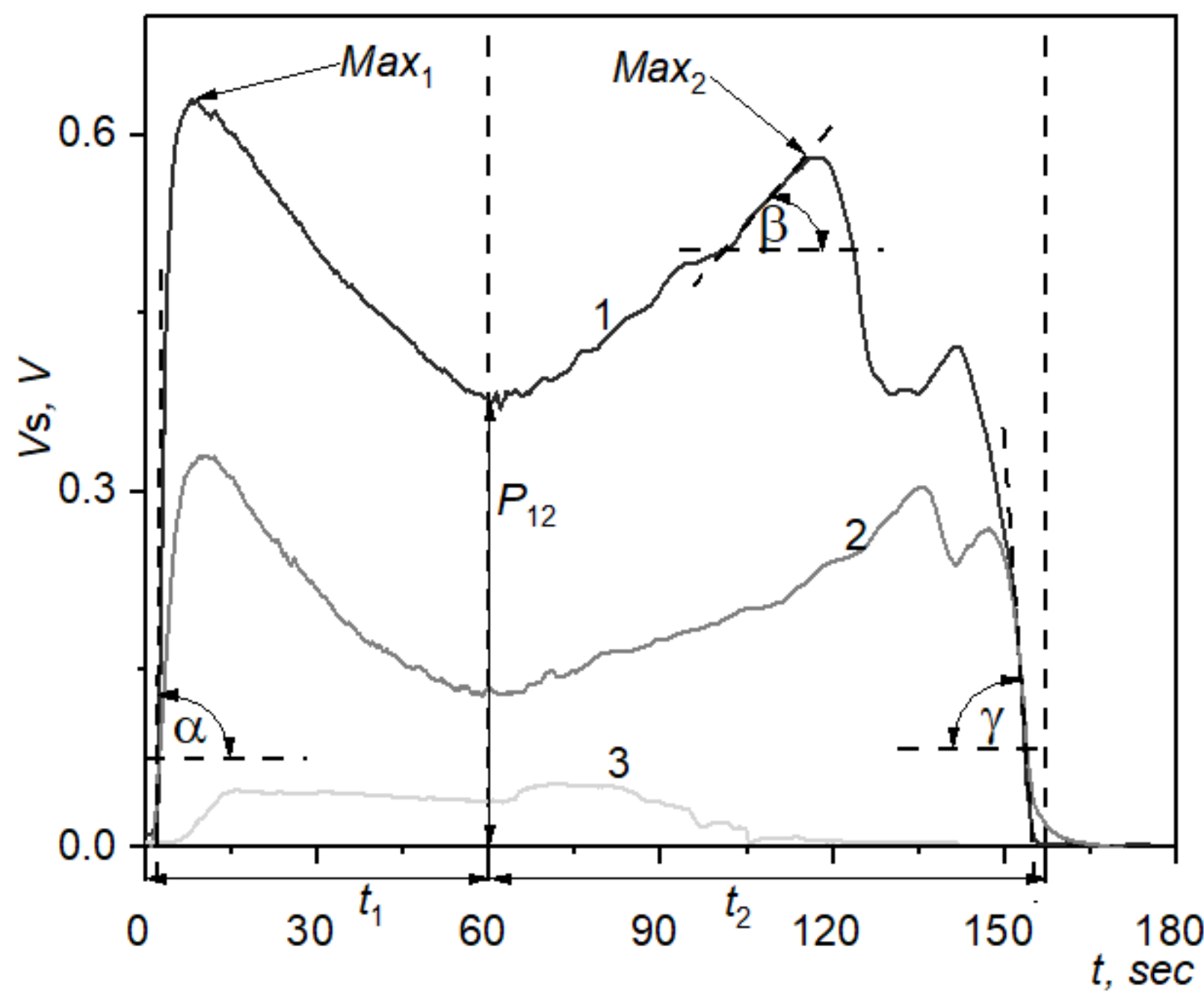

Figure 1 - Response curves of point-contact sensors based on TCNQ compound for the healthy person under the action of breath gas. $V_{\mathrm{s}}-$ voltage drop, which occurs in the sensor; $t$ - time; 1 response curve of the functional sensor with a typical response of high magnitude; 2 - response curve of the sensor of low magnitude, which was not be practically used; 3 - response curve of 
the sensor of extremely low magnitude and significant distortion of the response parameters.

Some characteristic parameters of the response curve (see, for example Ref. [19]): $t_{1}$ - exposure time; $t_{2}$-relaxation time; $\operatorname{Max}_{1}$ - maximum of the exposure period; Max $_{2}$ - maximum of the relaxation period; $p_{12}$ - ordinate of the final segment of the exposure phase; $d_{1}=\operatorname{tg} \alpha$ - slope of the initial part of the exposure peak; $d_{21}=\operatorname{tg} \beta$ - slope of the initial part of the most intense peak in the relaxation period; $d_{22}=\operatorname{tg} \gamma-$ slope of the final part of the most intense peak in the relaxation period;

It should be noted that breath gas is a highly composite system with a large amount of equilibrium and nonequilibrium dynamic interactions among the constituents. The components of such a rich gas mixture form in that way a certain breath profile, reflecting specific organism dysfunctions and metabolic disturbances. The analysis of breath can be performed through its profile which contains information about adsorption energies of the components of the gas mixture which can be considered to be in a quasi-equilibrium state at the moment of measurement. Due to quantum nature of their electric properties Yanson point contacts are able to record fine variations of superficial states of the point contact conductivity channel which are caused with adsorption of an external agent. The registration procedure is performed with the highest possible resolution of one conductivity quantum which is equal to addition of one atom to the point-contact channel $[28,29]$. This makes it possible to record response signals of a spectral type by point-contact sensors $[14,19]$. The characteristic parameters of the response curve, some of which are shown in Fig. 1 are used to describe the sensory profile of the breath gas and make it possible to find differences in the states of a healthy and diseased organism. The characteristic parameters of the point-contact sensor response curve and their application are described in detail in Refs. [19, 20, 26].

\section{Results and discussion}

To determine the relationship between the morphology of the obtained sensors and type of the sensor response curve observed under action of the human breath gas, a set of 3 different groups of sensors was used. The first group included sensors, the general view of the surface of which is shown in Fig. 2. They exhibit a typical response of large amplitude to the action of breath gas. The response curve of sensor device belonged to this group is shown as curve 1 in Fig. 1. It should be noted that based on the ease of practical use, we conditionally classified the levels of the response signal according to its maximum intensity. With a maximum signal intensity of more than $0.4 \mathrm{~V}$, the sensor was considered functional, since it had a response curve with large amplitude and features with good resolution. Such a response curve could be easily recorded and processed using a portable device [19, 20]. With a maximum response signal intensity of less than $0.4 \mathrm{~V}$ (Fig.1, curve 2), the sensors belonged to the group of samples with a low signal level (the second group in our case) and were not used for practical purposes. The general view of the surface of such samples is shown in Fig. 3. The third one consisted of samples (Fig. 4), which showed no or extremely low response to the action of the gas mixture (Fig.1, curve 3) that could be measured within the sensitivity of the recording devices. 


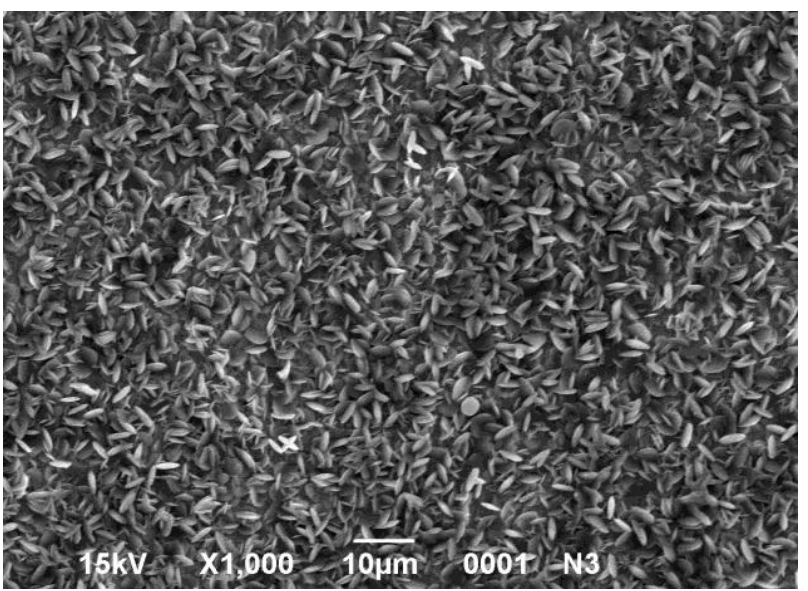

a

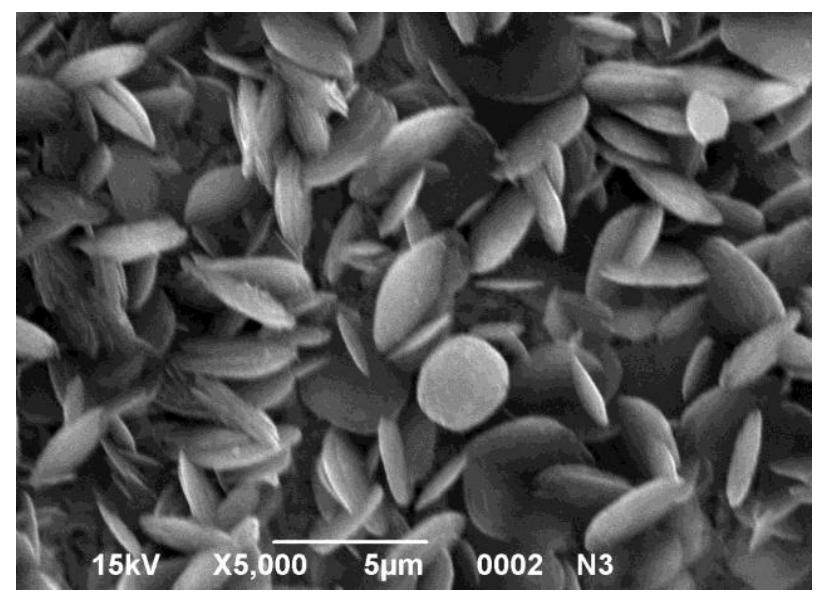

$\mathrm{b}$

Figure 2 - Micrographs of the sensitive surface of high-quality functional sensors at different scales.

As can be seen in Fig. 2, a high-quality functional sensor consists of a large amount of Yanson point contacts formed by layered $\mathrm{Cu}$-TCNQ crystals contacting each other. It follows from the above photographs that the crystals have a uniform shape, similar to a shell, and clearly defined external borders. The sensor sample is formed from crystals of the same or similar sizes. The crystal distribution is uniform over the surface of the sample. The scaly layered structure typical to the crystals of organic conductors can be seen [22,30]. The contacts are formed by synthetic crystals contacting each other without any visible distortion of their structure in the contact area. This fact indicates formation of defect-free Yanson point contacts in the process of soft electrochemical synthesis with a high degree of probability. And this, in turn, points to the possibility of them functioning under the conditions of a ballistic regime of current flow. As a result, the point contacts of this sensor array are able to work without destruction under conditions of high density current, which is confirmed by the high durability of these sensor samples in real usage [19]. It is found that there are about $5-6 * 10^{4}$ point contacts of this type for every square millimeter of a high-quality functional sensor surface. Thus, the surface of the sensor is a complex multistructure of a large number of Yanson point contacts, which generates a large output signal from the sensor in response to the action of the analyte. 


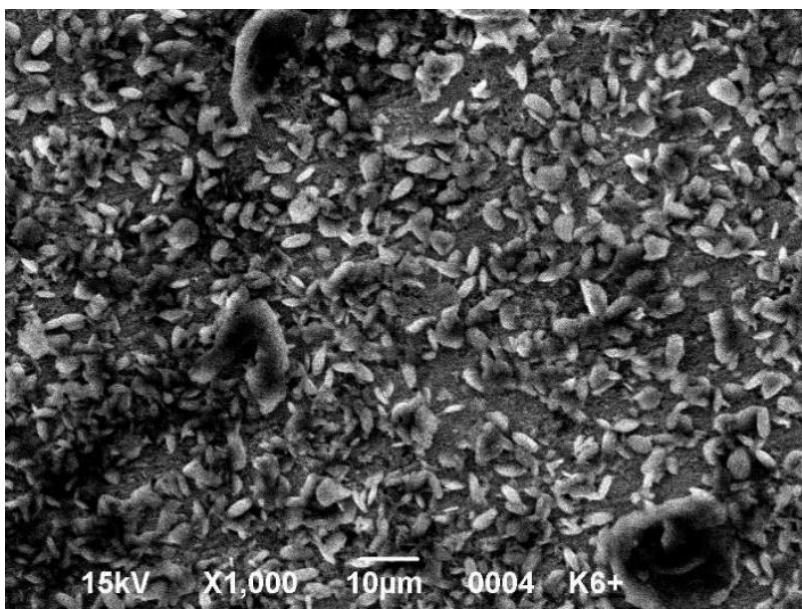

a

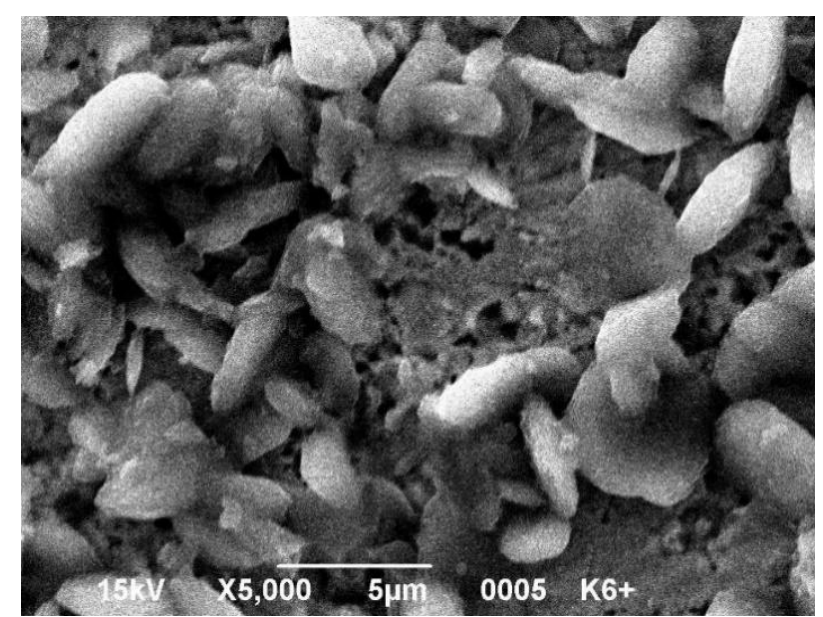

b

Figure 3 - Microphotographs of the sensitive surface of the sensor displaying a low-amplitude response.

Let us consider microphotographs of the sensors with a low level of useful signal. The response curve of sensor device belonged to this group is shown as curve 2 in Fig. 1. It can be seen from Fig. 3 that scaly layered $\mathrm{Cu}$-TCNQ crystals for sensors with a low response amplitude have a larger size and less uniformity than similar crystals for high-quality samples. Crystal boundaries are smeared and full of fractures. In some cases, contacts are formed on a large area. These structural features prevent the formation of high-quality Yanson point contacts. Contacts with this surface morphology most likely have distortions of the crystalline structure or other crystallographic defects, which reduces the mean free path of charge carriers [15]. This leads to an extra electron scattering, which reduces the magnitude of the useful signal caused by adsorption processes. In addition, the density of such crystals is also significantly lower, which, in turn, reduces the amount of functional contacts.

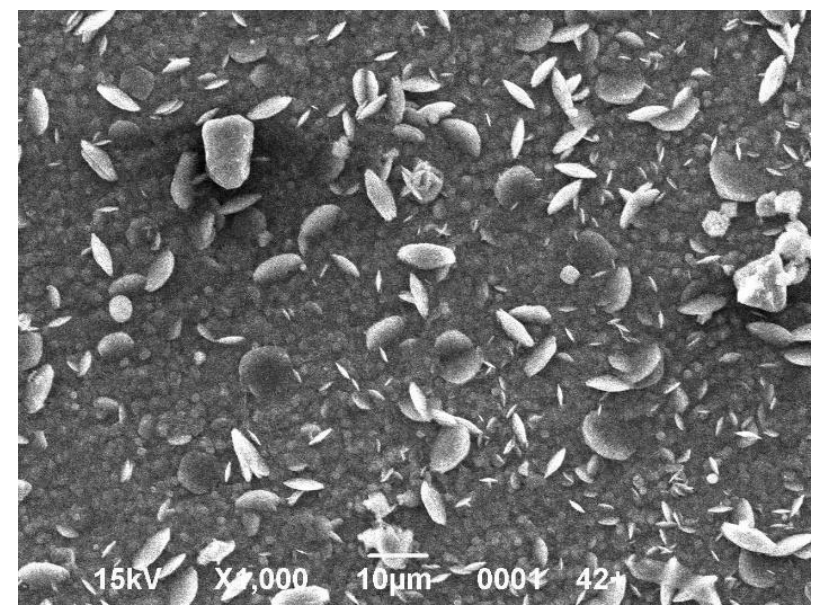

a

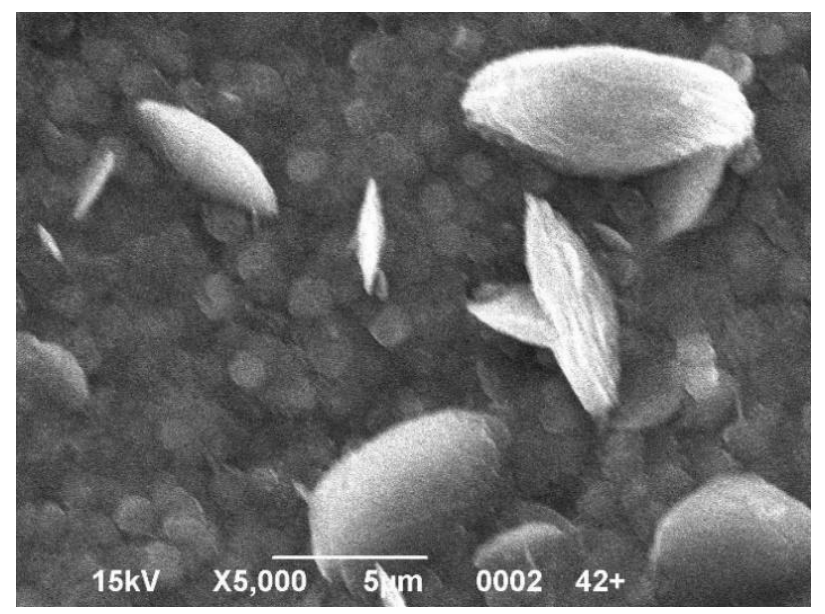

b

Figure 4 - Microphotographs of the sample surface showing no response to the action of breath gas which can be registered within the sensitivity of the available instruments. 
As can be seen in Fig. 4, crystals of Cu-TCNQ compounds for samples that do not show any response to the action of the gas medium have an extremely low density. It means that no sufficient number of point contacts and conducting channel can be formed between them. The response curve of sensor device belonged to this group is shown as curve 3 in Fig. 1.

Since the Cu-TCNQ compounds have a polymorphic nature [31], x-ray diffraction patterns were obtained to determine their phase composition. Figure 5 shows the range of angles $2 \theta=10-30^{\circ}$, in which the diffraction peaks of these compounds fall. In the present study, we used model samples deposited in regimes analogous to those used to obtain functional sensors and significantly different from them. It was found, however, that in all deposition regimes, the surface of the samples consisted of polycrystalline copper and the first phase of Cu-TCNQ compound, which implies that in real deposition regimes recrystallization into the second phase is unattainable.

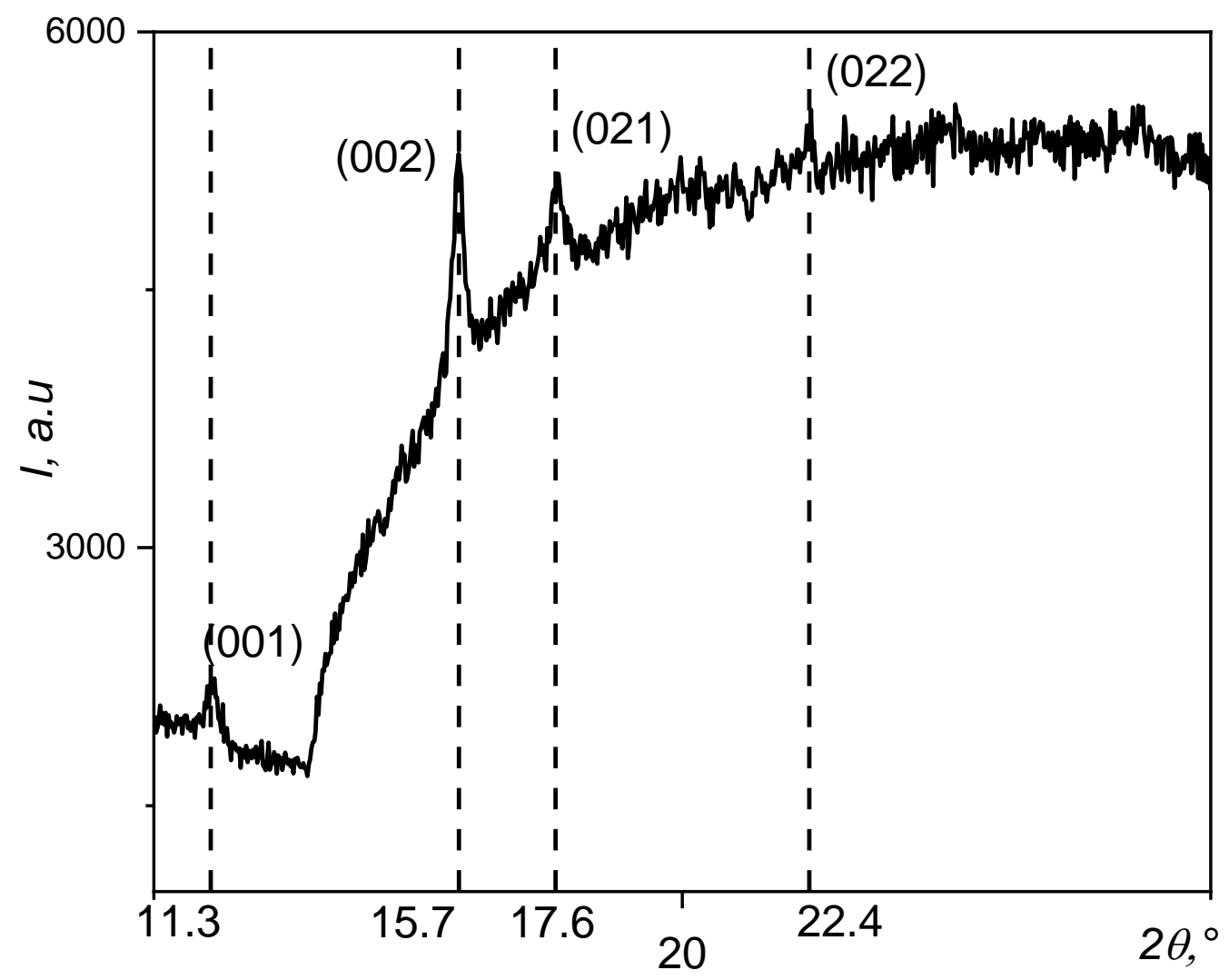

Figure 5 - XRD - pattern of model samples.

To determine the effect of various deposition regimes on the obtained surface morphology of the samples, several batches of model objects were obtained and their surface was studied. 


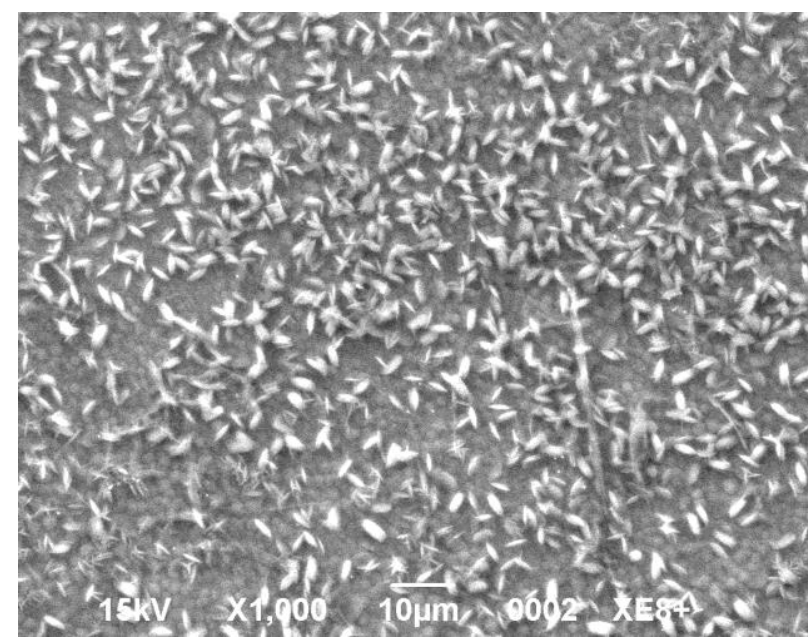

a

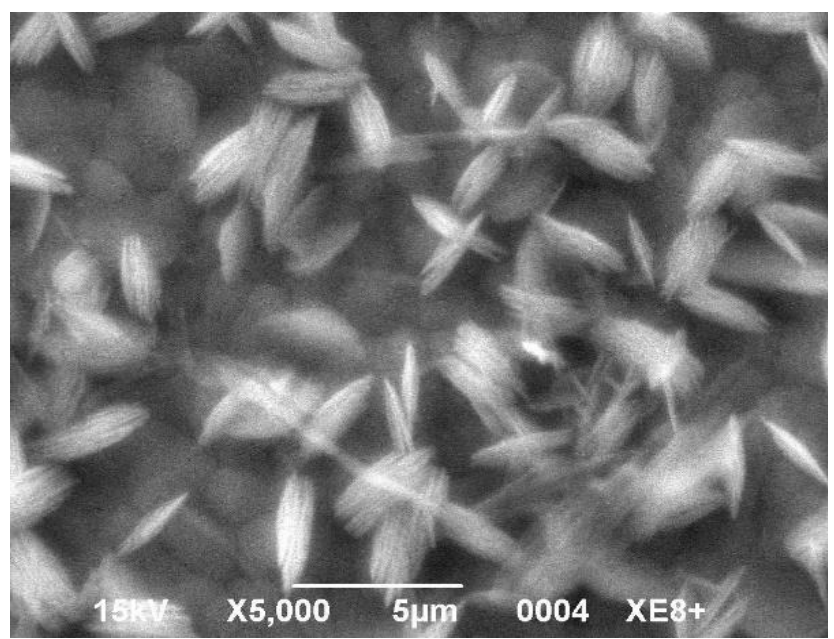

b

Figure 6 - A micrograph of the surface of a model sample obtained by combined

electrochemical deposition in a constant-voltage mode of $2.5 \mathrm{~V}$ for 7 minutes under forced convection regime.

As one can see in Fig. 6, for deposition regimes used to obtain model samples which were identical to that used to obtain functional objects a similar surface morphology was observed. A large number of layered Cu-TCNQ crystals of relatively low density are clearly visible. This may be due to the fact that the thickness of the copper foil used for the deposition of functional sensors is several orders of magnitude greater than the thickness of the copper film (300 A) used for the deposition of model samples.

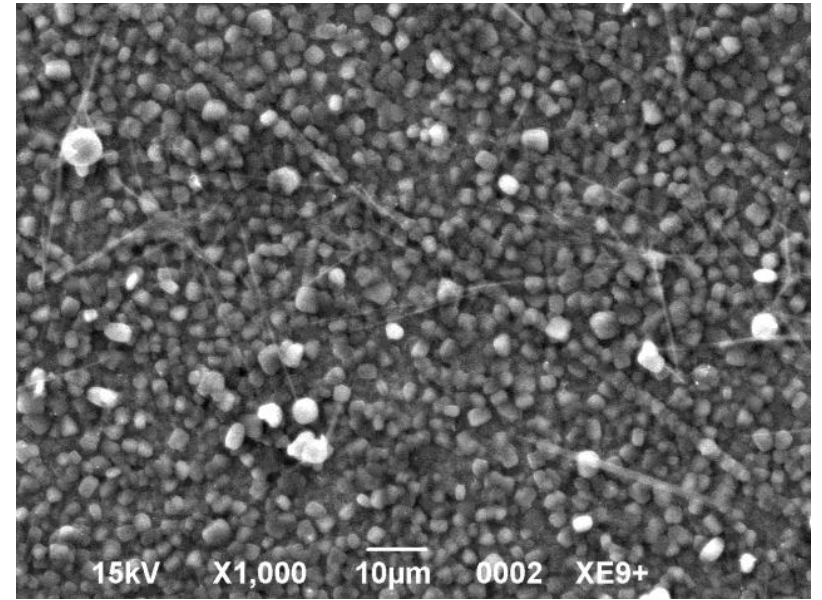

a

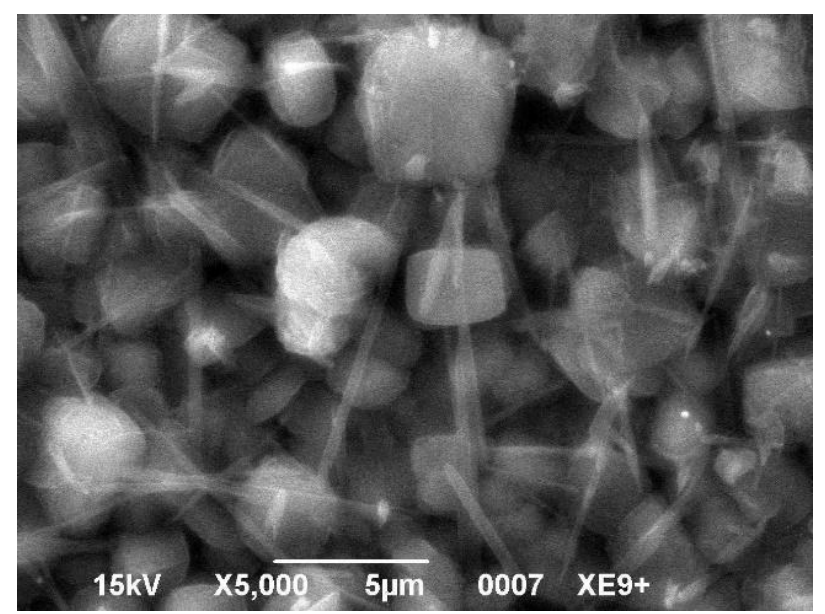

b

Figure 7 - A micrograph of the surface of a model sample obtained by combined electrochemical deposition in a constant-voltage mode of $2.5 \mathrm{~V}$ for 7 minutes without forced convection regime. 
As can be seen in Fig. 7, in the case of deposition of samples during the same time but in the absence of the forced convection, i.e., by pure electrochemical deposition, a drastically different surface morphology is formed. The crystals become larger; the layering is smoothed.

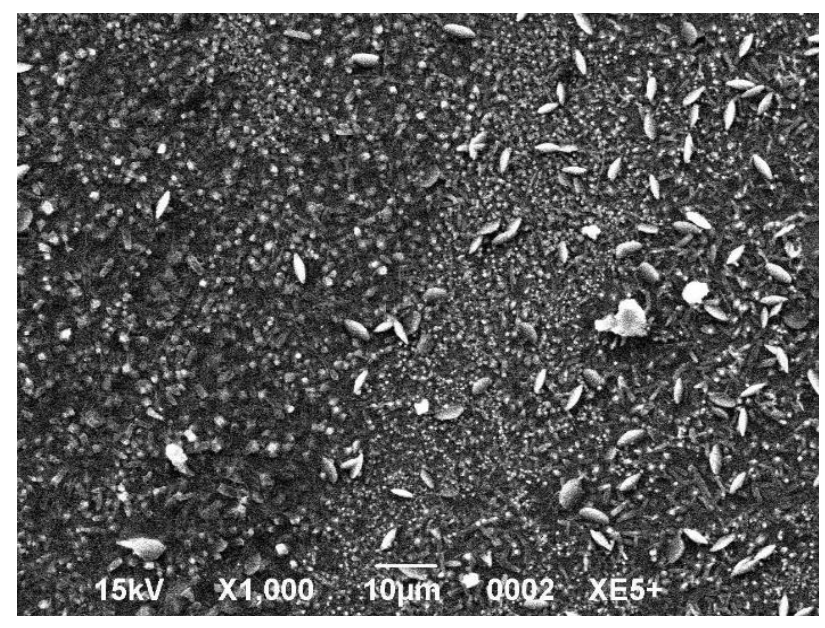

a

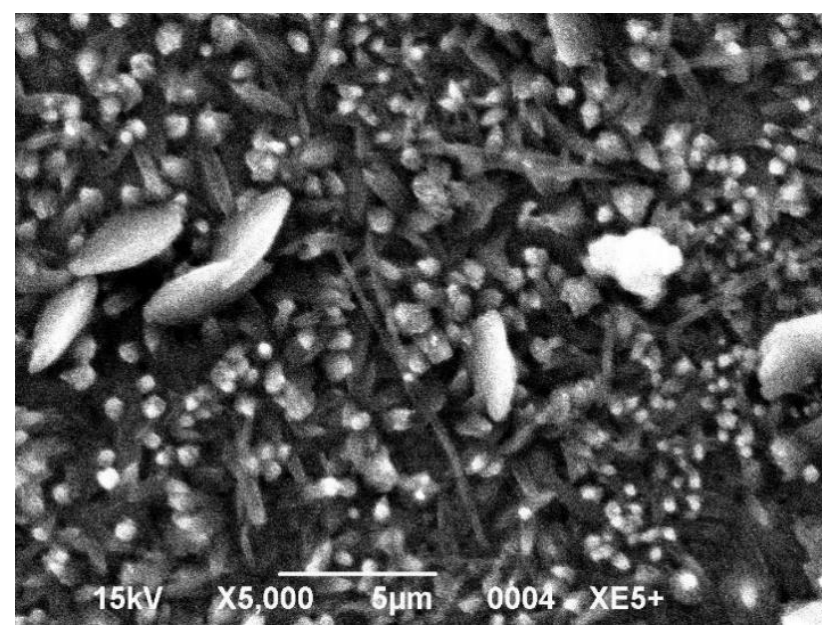

$\mathrm{b}$

Figure 8 - A micrograph of the surface of a model sample obtained by combined electrochemical deposition in a constant-voltage mode of $2.5 \mathrm{~V}$ for 5 minutes under conditions of forced convection.

As can be seen in Fig. 8, with a decrease in the deposition time, the number of scaly crystals decreases and smaller crystallites occupy a large part of the surface. It is found that sensors with a similar surface morphology of the gas sensitive layer have low response amplitude when exposed to the gas medium.

\section{Conclusion}

The phase composition and surface morphology of the obtained sensor films were established. It was found correlation between surface morphology of the point-contact sensor and definite type of the sensor response curve observed under action of the human breath gas. A high-quality functional sensor consists of a large amount of Yanson point contacts formed by layered $\mathrm{Cu}$-TCNQ crystals contacting each other. Numerous Yanson point contacts are combined in a complex multistructure which generates a large output signal from the sensor in response to the action of the analyte. A decrease in the density of $\mathrm{Cu}-\mathrm{TCNQ}$ crystals leads to a decrease in the concentration of Yanson point contacts in the sensor multistructure and, ultimately, to a decrease in the intensity of its output signal. The studies performed in this work allow us to improve the understanding of the nature of point-contact sensors based on TCNQ compounds and create the prerequisites and opportunities for their wide practical use.

\section{Acknowledgements}

This work was partly supported by the NATO SPS Programme (Ref: SPS 985481). 


\section{References}

1. Matushko, I.P., Arinarkhova, H.O., Oleksenko, L.P., Maksymovych, N.P., \& Fedorenko, G.V., Nanosized materials $\mathrm{CeO} 2 / \mathrm{SnO} 2$ and $\mathrm{CeO} / \mathrm{SnO} 2-\mathrm{Sb} 2 \mathrm{O} 5$ for adsorption semiconductor sensors to detect hydrogen in air. Molecular Crystals and Liquid Crystals, 2018. 673(1): p. 23-31.

2. Nespurek, S., Mracek, L., Kubersky, P., Syrovy, T., \& Hamacek, A., lonic liquids in electrochemical gas sensors and transistors. Molecular Crystals and Liquid Crystals, 2019. 694(1): p. 1-20.

3. Nahirniak, S.V., Dontsova, T.A., \& Chen, Q., Sensing properties of SnO2-MWCNTS nanocomposites towards H2. Molecular Crystals and Liquid Crystals, 2018. 674(1): p. 48-58.

4. Schroeder, V., Savagatrup, S., He, M., Lin, S., and Swager, T. M. , Carbon Nanotube Chemical Sensors. Chemical Reviews, 2019. 119(1): p. 599-663.

5. Kong, J., Franklin, N.R., Zhou, C., Chapline, M.G., Peng, S., Cho, K., and Dai, H., Nanotube molecular wires as chemical sensors. Science, 2000. 287(5453): p. 622-625.

6. Karachevtsev, M.V., Gladchenko, G. O., Plokhotnichenko, A. M., Leontiev, V. S. and Karachevtsev, V. A., Adsorption of biopolymers on SWCNT: ordered poly $(r C)$ and disordered poly(rl). Journal of Physical Chemistry B, 2013. 117(9): p. 2636-2644.

7. Kamarchuk, G.V., Kolobov, I.G., Khotkevich, A.V., Yanson, I.K., Pospelov, A.P., Levitsky, I.A., and Euler, W.B., New chemical sensors based on point heterocontact between single wall carbon nanotubes and gold wires. Sensors and Actuators B, 2008. 134(2): p. 1022-1026.

8. Kamarchuk, G.V., Pospelov, A.P., Kushch, I.G., Sensors for exhaled gas analysis: an analytical review, in Volatile biomarkers: non-invasive diagnosis in physiology and medicine, A.Amann and D.Smith, Editors. 2013, Elsevier: Amsterdam. p. 265-300.

9. Li, W., Liu, H., Xie, D., He, Z., \& Pi, X., Lung Cancer Screening Based on Type-different Sensor Arrays. Sci Rep 2017.7 (1969 (2017)): p. 12 pp.

10. Costello, B.d.L., Amann, A., Al-Kateb, H., Flynn, C., Filipiak, W., Khalid, T., Osborne, D. and Ratcliffe, N. M., A review of the volatiles from the healthy human body. J. Breath Res., 2014. 8(1): p. 014001 (29 pp).

11. A. Amann and D. Smith, eds. Volatile biomarkers: non-invasive diagnosis in physiology and medicine. 2013, Elsevier: Amsterdam. 570.

12. Montuschi, P., Mores, N., Trove, A., Mondino, C. \& Barnes, P. J. , The electronic nose in respiratory medicine. Respiration, 2013. 85(1): p. 72-84.

13. Szulczynski, B., Namiesnik, J, and Gebicki, J., Determination of Odour Interactions of ThreeComponent Gas Mixtures Using an Electronic Nose. Sensors, October 2017. 17(10 ): p. 2380 (18 pp).

14. Kamarchuk, G.V., Pospelov, A.P., Kamarchuk, L.V., Savytskyi, A.V., Harbuz, D.A., Vakula, V.L., Point-Contact Sensors as an Innovative Tool in Defense Against Chemical Agents, Environment and Health Risks: A Review, in Functional Nanostructures and Sensors for CBRN Defence and Environmental Safety and Security A. Sidorenko, Hahn, H., Editor. 2020, Springer: Dordrecht. p. 245-270.

15. Yu.G. Naidyuk and I.K. Yanson, Point-contact spectroscopy. 2005, New York: Springer. 300. 
16. A.V. Khotkevich and I.K. Yanson, Atlas of Point Contact Spectra of Electron-Phonon Interactions in Metals. 1995, Boston/Dordrecht/London: Kluwer Academic Publishers. 168.

17. Kamarchuk, G.V., Pospelov, O.P., Yeremenko, A.V., Faulques, E., Yanson, I.K., Point-Contact Sensors: New Prospects for a Nanoscale Sensitive Technique. Europhys. Lett., 2006. 76(4): p. 575-581.

18. Kamarchuk, G.V., Pospelov, A.P., Kamarchuk,L.V., and Kushch, I.G. , Point-Contact Sensors and Their Medical Applications for Breath Analysis: A Review, in Nanobiophysics: Fundamentals and Applications, V.A. Karachevtsev, Editor. 2015, Pan Stanford Publishing Pte. Ltd. p. Chapter 11, p. 327-379.

19. Kushch, I., Korenev, N., Kamarchuk, L., Pospelov, A., Kravchenko, A., Bajenov, L., Kabulov, M., Amann, A., Kamarchuk, G., On the importance of developing a new generation of breath tests for Helicobacter pylori detection. J. Breath Res., 2015. 9(4): p. 047109 (15 pp).

20. Kamarchuk, G.V., Pospelov, A.P., Harbuz, D.A., Gudimenko, V. A., Kamarchuk, L.V., Zaika, A.S. , Pletnev, A.M., Kravchenko,A.V., Nanostructural point-contact sensors for diagnostics of carcinogenic strains of Helicobacter pylori. Biophysical Bulletin, 2017. 2(38): p. 66-78.

21. Golovko, S.A., Gudimenko, V.A. , Klimkin, A.S., Pletnev, A.M., Vakula, V.L., Zaika, A.S., Kamarchuk, L.V., Kushch, I.G., Pospelov, A.P., Kravchenko, A.V., Kamarchuk, G.V., Development of Criteria for Analysis of Point-Contact Sensor Characteristics in Complex Gas Media. Universal Journal of Materials Science, 2016. 4(2): p. 32-39.

22. Kravchenko, A.V., Starodub, V.A., Kazachkov, A.R., Khotkevich, A.V., Pyshkin, O.S., Kamarchuk, G.V., Spectral and electrophysical characteristics of anion-radical salts of TCNQ and methylTCNQ with N-alkylpirazinium cations, in Spectroscopy of Emerging Materials, E.C. Faulques, Perry D.L., and Yeremenko, A.V., Editor. 2004, Kluwer Academic Publishers, NATO Science Series: Boston/Dordrecht/London. p. 319-330.

23. Pyshkin, O., Kamarchuk, G., Yeremenko, A., Kravchenko, A., Pospelov, A., Alexandrov, Yu., and Faulques, E., Evidence for sensory effects of a 1D organic conductor under gas exposure. J. Breath Res., 2011. 5(1): p. 016005 (9 pp).

24. Chubov, P.N., I.K. Yanson, Akimenko, A.I., Electron-phonon interaction in aluminum point contacts. Fizika Nizkikh Temperatur, 1982. 8(1): p. 64-80.

25. Potember, R.S., Poehler, T. O., \& Cowan, D. O., Electrical switching and memory phenomena in Cu-TCNQ thin films. Appl. Phys. Lett. , 1979. 34: p. 405-407.

26. Kushch, I.G., Korenev, N.M., Kamarchuk, L.V., Pospelov, A.P., Alexandrov, Y.L., and Kamarchuk, G.V., Sensors for Breath Analysis: An Advanced Approach to Express Diagnostics and Monitoring of Human Diseases, in Biodefence. NATO Science for Peace and Security Series A: Chemistry and Biology, A.K. S. Mikhalovsky, Editor. 2011, Springer Science+Business Media B. V., chapter 7. p. 63-75.

27. Kamarchuk, L.V., Pospelov, A.P., Harbuz, D.A., Belan, V.I., Volkova, Yu.V., Tkachenko, A.A., Kamarchuk, G.V. , Noninvasive real-time breath test for controlling hormonal background of the human body: Detection of serotonin and melatonin. to be published.

28. Pospelov, A.P., Pilipenko, A. I., Kamarchuk, G. V., Fisun, V. V., Yanson, I. K., and Faulques, E., A New Method for Controlling the Quantized Growth of Dendritic Nanoscale Point Contacts via Switchover and Shell Effects. J. Phys. Chem. C, 2015. 119(1): p. 632-639.

29. Kamarchuk, G.V., Pospelov, A.P., Savytskyi, A.V., Herus, A.O., Doronin, Yu.S., Vakula, V.L., Faulques, E., Conductance quantization as a new selective sensing mechanism in dendritic point contacts. SN Applied Sciences, 2019. 1:244.

30. R.P.Shibaeva and E.B.Yagubskii, Molecular Conductors and Superconductors Based on Trihalides of BEDT-TTF and Some of Its Analogues. Chem. Rev., 2004. 104(11): p. 5347-5378. 
31. Ramesh, P., \& Srivastava, D., Distinguishing Polymorphs of Copper- Tetracyanoquinodimethane charge-Transfer Salts. Science and Technology Journal, 2015. 3: p. 125-133. 